\title{
Design of Pneumogram for Chronic Obstructive Pulmonary Disease Diagnosis
}

\author{
D.K. Ravish, K.J. Shanthi, Nayana R Shenoy
}

\begin{abstract}
The cessation of breathing is a common initial symptom of many of distinct diseases. Reliable and obtaining right information timely is a strategic advantage in identification of congenital anomalies. Often this makes the significant difference between an infant's survival and death. Such respiratory device defects also illustrate how physicians must carefully choose among many detection modalities available to most effectively and appropriately arrive at a diagnosis and treatment. The objective of the work is study of comparative safe pnemogram device usages with high accuracy and also to obtain robust clinical information. The impedance pneumogram (IP) and acoustic pnemogram (AP) a hybrid model is presented here could be useful to clinician.
\end{abstract}

Keywords: Pneumogram, Acoustic, Pediatric, Pulmonary disease, respiration rate.

\section{INTRODUCTION}

Chronic obstructive pulmonary disease (COPD) is a main agency of fatality and anguish worldwide with an approximated 22 million deaths in 2016, based on the reports by world health organization (WHO) and fourth noted cause of death. In inclusion to the considerable fatality and anguish related with COPD, this ailment arouses important clinical and societal costs. Current COPD ground rule endorse that the following can improve COPD deaths: smoking cessation in adults; continuing oxygen therapy; and lung volume diminution surgery in little subgroup of COPD subjects. To date, no random inhibited trials have validated an outcome of pharmacological therapy on fatality, admitting several experimental trials justify that the pair long-acting bronchodilators and inhaled corticosteroids may give a survival benefit.

\section{Respiration}

First, Respiration is an act of breathing. Respiration is exchange of gases in biological process. In our body, the respiratory mechanism is viewed as pneumatic system. A physiological air pump called diaphragm creates alternatively negative and positive pressures in a chamber called thorax. The air to be sucked into and out of a pair of lungs within the thoracic cavity. The lungs are connected to the surrounding air medium through nasal cavities has a common pathway for carrying air cavity air to lungs and food to stomach. A special valve blocks the airway whenever food passes through the common region. Inhalation process (inspiration) of flowing air into lungs, the muscle will contract so that the ribs will lift and pull outward to increase lung volume. The increase in lung volume allows air to be rushed to lungs and this is active process. The inspiration air must contain at least 21 percent of oxygen. The exhaling (expiration) is a passive process sometimes it is termed as reverse process of flowing out or air outing. The decrease in lung volume will exhaust the air out of lungs. The oxygen remnant still present in exhausted air is about 16 percent and the traces of other gases too present in it. The respiration system works on the inspiratory reflex. Respiratory rate depends on the various parameters like i) concentration of gases ii) lung tissue pressure iii) decrease in blood pressure and change in $\mathrm{pH}$ value in body fluid.

\section{Respiratory mechanism}

The respiratory system consists of two tracts. The one is named as upper respiratory tract; this consists of nasal that is termed here as upper cavity, sinuses, mouth, and larynx and windpipe trachea. The other is lower tract which includes the lungs, bronchi and alveoli. A human respiratory system is shown in Figure. The two lungs, one on the right and the other on the left, are the body's major respiratory organs. Each lung is divided into upper and lower lobes. But a third division is right lung called right middle lobe. And hence right lung is larger and heavier than left. The parts of the human respiratory system are shown in Figure.

The reason of smaller size comparatively to right lung is left space occupied by the heart. A shiny, clear, thin coating called the pleura envelops the lungs. The inner visceral layer wet surface between two glass slides sticks like the glass plates together. Similarly a pleural fluid between visceral layer and parietal layer keeps both layers in place. Mediastinum is an area that contains the heart and its large vessels, the trachea (windpipe), esophagus, thymus, and lymph nodes. The diaphragm made up of muscle dissects the thoracic cavity from the visceral cavity. The main function of diaphragm is contracting, relaxing to perform breathing.

Revised Manuscript Received on August 14, 2019.

D.K. Ravish, Department of Medical Electronics, Dr.Ambedkar Institute of Technology, Bangalore, Karnataka, India. (Email: ravishait@yahoo.co.in)

K.J.Shanthi, Department of Medical Electronics, Dr.Ambedkar Institute of Technology, Bangalore, Karnataka, India. (Email: shantikj@yahoo.co.in )

Nayana R Shenoy Department of Medical Electronics, Dr.Ambedkar Institute of Technology, Bangalore, Karnataka, India. (Email: nayana.rshenoy@gmail.com) 


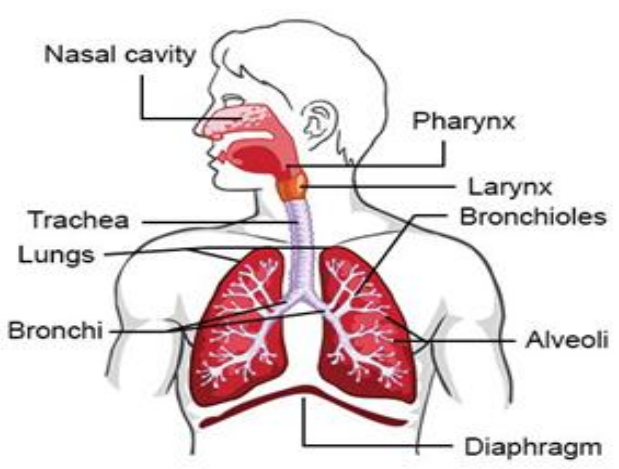

Fig.1 Human Respiratory System Source: pinterest 2018.

\section{LTERATURE REVIEW}

Monitoring respiration could find oxygenation and ventilation complications early before cardiac arrest and neurological arrests [1]. An abnormal respiratory measurement is a conscious predictive warning of critical illness [2]. Pulmonary disorder is an originator of ill health throughout the world. In Europe chronic obstructive pulmonary disease as asthma has been predicted to upset between $10-20 \%$ of the adult people. Lung diseases such as intense bronchitis, pneumonia are frequent and interstitial lung abnormality is rise in incidence. Detection of these frequent respiratory illnesses is expedited by respiratory organ auscultation sound using stethoscope. This medical instrument is invented in 1821 by the French inventor, Laennec, is still the accepted distinguished tool used by the clinicians. Listening to the sounds with device, stethoscope has many constraints. It is the clinician dependent process that depends on the doctor's own hearing. It is subjective criteria and capability of one's discerning or indentifying of variant sound patterns. It is not easy to yield quantitative measurement or make an invariable record of a test in documentary form.

Medical instrument stethoscope has many limitations; one important drawback is frequency bands. The respiratory signals mostly of low frequencies lies within the band of $125 \mathrm{~Hz}$. Human ear is insensitive to the lower frequency range. The other constraints are its amplification is through mechanical tube like structure and diaphragm based on the principles of sound amplification by day old conventional medical physics. Moreover acuity of listening to the sound by human is excellent at $1000 \mathrm{~Hz}$ and certainly low frequencies human has having difficulty in listening to a sound and having higher hearing threshold. The stethoscope sound is not much perceptive. Over the last three decades, use of computer methods and digital signal processing applications in the bio signal advancements occurred Acquisition and analysis of respiratory sounds overcomes many restrictions of simple auscultation [4]. Present years the recent activity in respiratory signal acquisition and analysis has increased remarkably.

\section{DESIGN METHODOLOGY}

\section{A. Recording of respiratory sounds}

Patient data: The gender of the subjects and their alternate basis utilized has to be determined. The smoking history of the subject precondition (for adult subjects) be known if it so. In all the subjects' specific information anthropometric must be understood first (pediatrics). The readiness of the subjects' previously assessment must be noted: smoking, eating, drinking and specially drug in take. The detail knowledge about treatment undergone for asthma (chronic) is of significant indicator.

Surroundings: When the regular frequency maximums of existence noise due to noise from outside source have not been prevented, the overlap of signal frequency components and environmental surrounding sound also required to study.

The accuracy of measurement depending on the accuracy of: Recording device, amplifier, signal processing methods and analysis. Practical assessment: Body aspect, the condition of alertness (Hypnos studies), and route of respiration during recording have to be characterized.

\section{B. Sound acquisition system:}

\section{i) Microphone fixation-}

The positioning of microphone during sound acquiring has to be defined. Sometimes, it is reasonable to record lung sound on the location where to breathe sounds adventitious lung sounds are best heard.

\section{ii) Microphone sensors}

the types of sensor available are contact microphone sensor, as shown in Figure 2 (condenser or piezoelectric microphones). The calibration procedures of the sensor and the frequency response in actual use have to be determined. If several sensors are used, a balancing procedure between the sensors has to be recorded.

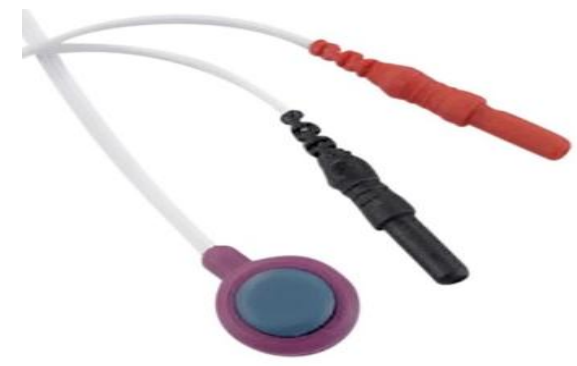

Fig 2. Pick-up sensor.

Source: Ambu products 2018

iii) Sound recording:

Analog recording signal is stored in digital format using analog to digital conversion method using data acquisition card with suitable sampling frequency $8000 \mathrm{~Hz}$ is adequate [5].

\section{iv) Amplifier:}

Amplification gain for respiratory signal specifications are required to be in the low frequency range up equate as literatures discusses $2000 \mathrm{~Hz}, 8000 \mathrm{~Hz}$ and a gain of 500 is sufficient. Analog devices amplifier AD 620 is used in this application in the pre-processing stage. Amplifier circuit is implementing for amplification purpose is represented in Figure 3. 


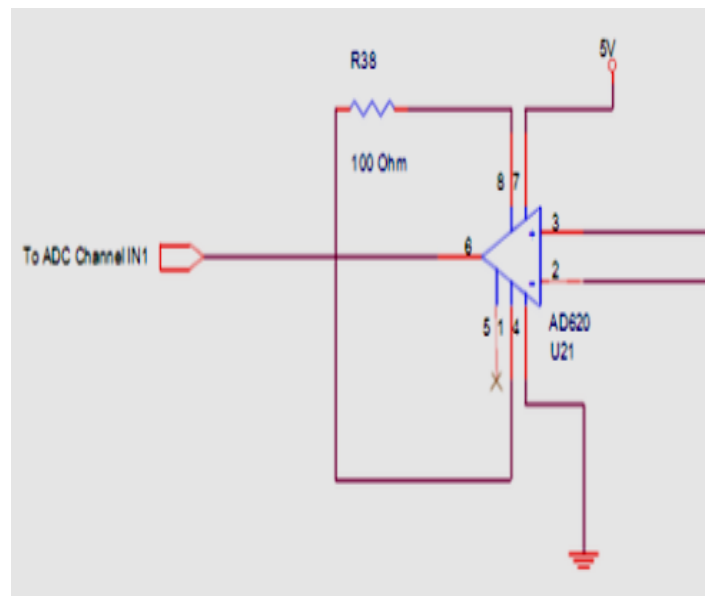

Fig 3. Amplifier circuit using AD 620.

\section{Source: AD instruments 2018}

\section{v) Sound optimizing:}

The cut-off frequency of high pass filter (HPF) or low pass filter (LPF), that is the frequency at which thousand or subset of the signal is reduced $3 \mathrm{~dB}$, and the roll of factor (ROF) of the signal optimizing ( in $\mathrm{dB}$ - Octave 1) in accordance with the requirements. As it is desired to signal to be viewed and analyzed in real-time, here Butterworth filter properties are most suitable one. The Butterworth filter designed to have frequency of band of $0.5 \mathrm{~Hz}$ (DC) to 5000 $\mathrm{Hz}$ on the linear amplitude scale.

v) The frequency of maximum intensity (Fmax) and the lung sound amplitude in terms of the root mean square (RMS) value of the total power spectrum are also computed within the same analyzing band [6].

\section{vi) Sound Analysis:}

Extraction of signal for analysis be marked. Entire recording choosing criteria include inspired or expiratory signal by some definite reporting.

\section{Time domain analysis:}

In breath sound determination of events with respect to time duration presentation of signal is very adventitious. Breathing signals of examples for normal and abnormal real time signals are shown in Figure 4.

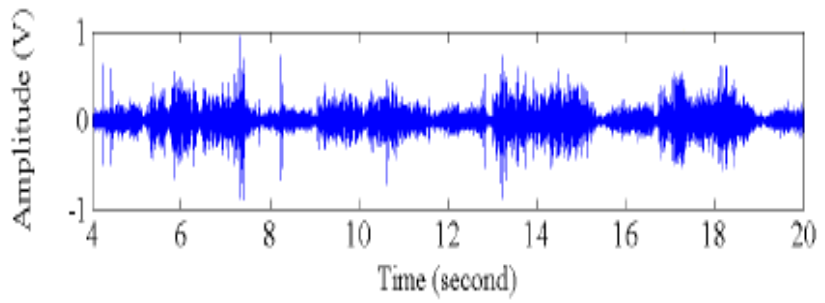

(a) Normal breathing signal

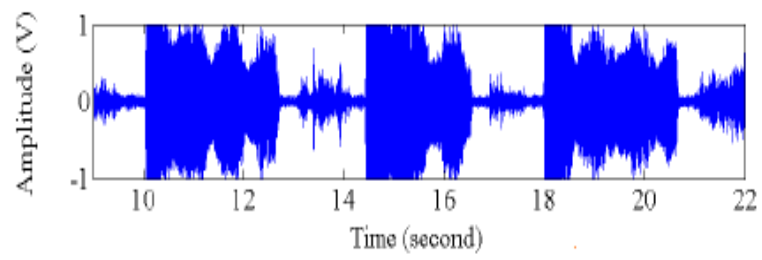

(b) Wheezing breathing signal

Fig 4. Exemplary real time breathing signals

\section{Morphological analysis:}

Waveform morphology understanding and detection of crackles and wheezes.

\section{E. Spectral analysis:}

Define the linear phase (stable) windowing method moving averaging model (MA filter) fast Fourier Transform (FFT algorithm) is applied to signal to estimate spectral power as well frequency components. These features are useful in discerning normal respiratory signal and diseased conditions.

\section{IMPLEMENTATION \& RESULTS}

a. Impedance pneumography:

It is an indirect method and does not require any placement of mask on the face (cannula), fixing of tubes flow meters and/or spirometers either in the nose or in the mouth. In this method, an electrode is placed externally over the thorax region and hence does not give any physiological effect on the patients. The schema of method of represented in Figure 7.

The electrode used here is a self adhesive type. The motion artifacts can be minimized by having an electrode cream layer. The skin resistance will be in the range of 150$200 \mathrm{Ohm}$. The change in resistance will be in the order of 1 percent of base resistance. A high frequency signal will be applied across the resistance and the potential drop across the resistance will be taken and applied across a high input impedance amplifier. A demodulator and filter will give a magnitude proportional to the change in the resistance of the electrode. This magnitude is calibrated to read the respiratory rate directly. This method gives faithful output and ease of use for quite patients. The disadvantage of this method is it gives more artifacts for (ambulatory) moving patients. Artifact free care must be taken while implementing on children.

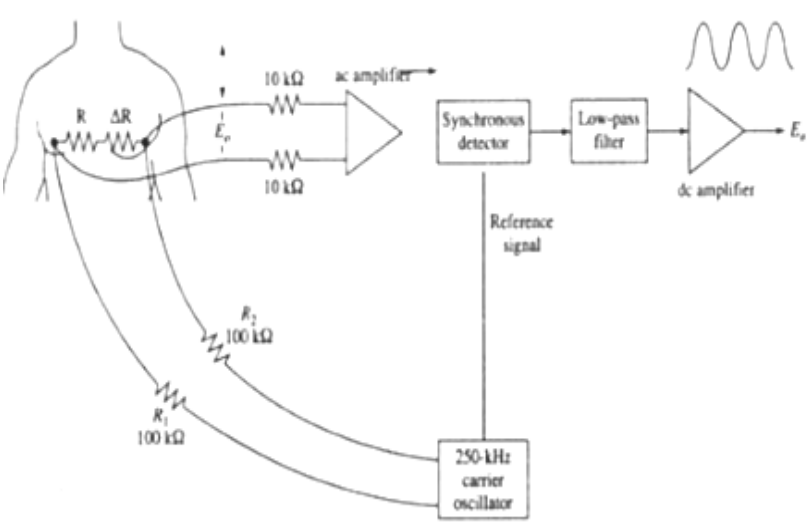

Fig 5. Amplifier circuit using AD 620.

Source: AD instruments 2018

The impedance pneumograph is a bio impedance recorder for indirect method of measurement airway resistances during respiration process. Electrodes Placement on to the chest, the impedance pneumograph measures respiratory 
tidal volume as well respiration rate through the relationship is a non invasive technique used for continuous and dynamic measurement of respiratory volume and rate. The impedance pneumography employs low amplitude, high frequency Alternating current passed through electrodes to record the thoracic movements or volume changes at rib cage during a respiratory cycle. Based on ohm's law, the voltage drop across the electrodes is computed as change in impedances. There is change in the impedance of the skin of thoracic region as shown in Figure 6. The impedance increases during inspiration and decreases during expiration, the mechanism of breathing is depicted in Figure 6.

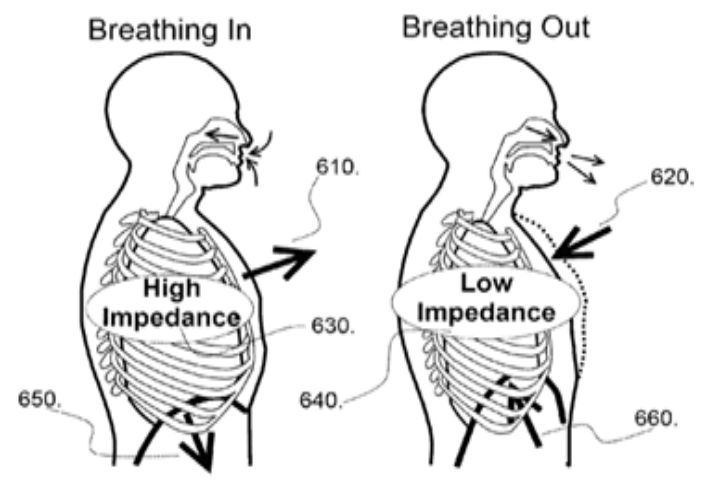

Figure 6. Impedance produced during respiration.

\section{B. Description of working principles:}

The impedance measurement is commonly performed using one of two primary electrode configurations: the tetra polar configuration or the bipolar configuration. Here the tetra polar-electrode configuration is used as it generally provides a more uniform current density and reduces the effect of skin-electrode impedance in the sensed voltage. The block diagram of the system and method are shown in Figure 7.

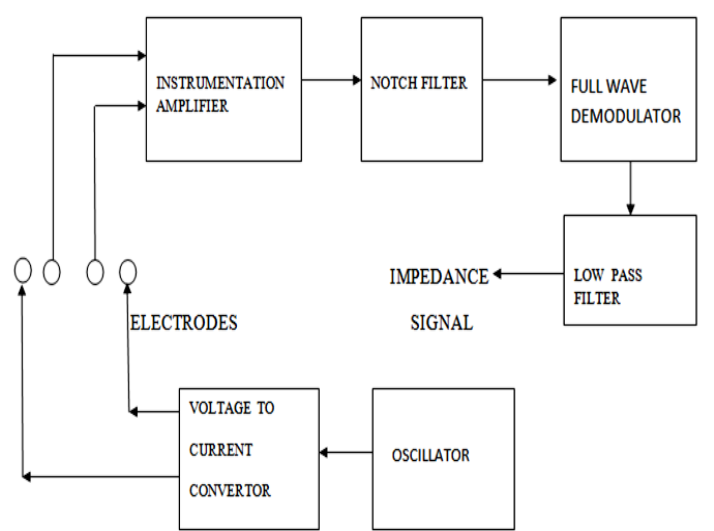

Fig 7. Block diagram of impedance pneumography system.

The oscillator is used for generating a sinusoidal signal with 50 to $500 \mathrm{kHz}$ frequency. The higher frequencies avoid shock to the body when current injected. The current is injected via two application electrodes (inner electrodes) and the voltage is detected via the sensing electrodes (outer electrodes). The outer pair transmits the ac current and detects the changes in the impedance due to working of the lungs. The voltage resulting across the body segment, between respiratory depth and thoracic impedance change. It

sensed using the inner electrodes, gets amplitude modulated due to the impedance variation.

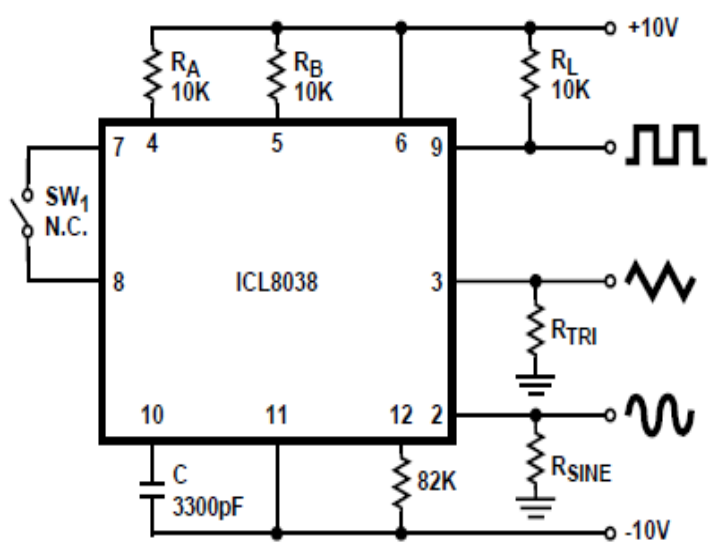

Fig 8. High frequency oscillator circuit.

The signal is then passed to the instrumentation amplifier as the signal generated from the body will be of very low amplitude, so the instrumentation amplifier amplifies it. This signal is then passed to $50 \mathrm{~Hz}$ narrow band reject filter to removal of the power line noise. As the voltage signal gets amplitude modulated due to impedance variation this voltage is demodulated using an envelope detector to get back the original signal. As the respiration rate frequency is of very low between 0.0 to $0.5 \mathrm{~Hz}$, the low pass filter was used to eliminate the high frequency components. The impedance can be approximated by the simple application of the Ohm's law. In order to avoid any physiological effects, a low-level current $(<5 \mathrm{~mA})$ in the frequency range of $20 \mathrm{kHz}$ to $1 \mathrm{MHz}$ is used. In this frequency range, the tissues are not excitable (except possibly at very high current levels) and the impedance is nearly resistive. The impedance values, from several measurements over time, are presented as an impedance waveform. The circuit proposed for the impedance measurement is given in Figure 8. Thus through the impedance signal, respiration peaks are detected. A typical impedance pnemogram signal is shown in the Figure 9. Signal $x$-axis showing number of respirations and in $y$ axis magnitude proportional to change in impedance level is depicted.

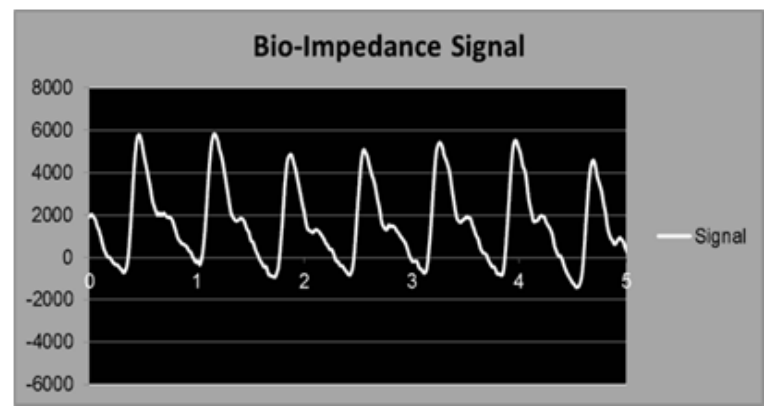

Fig 9. Bio impedance signal.

\section{RESULT AND CONCLUSION}

The beginning is what electrode arrangement would be the leading and how to connect them to skin. Afterwards, 
which places of connection may give us the high fidelity signal, and decisively, in what conditions there are more artifacts and how to extract the signal even when we do not have the potentiality to get the thoracic impedance signal.

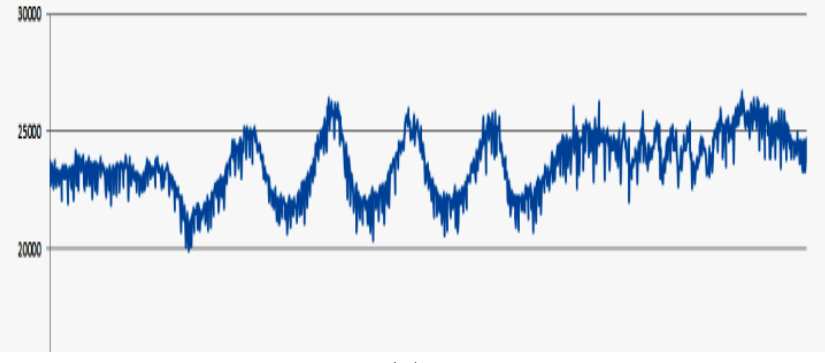

(a)

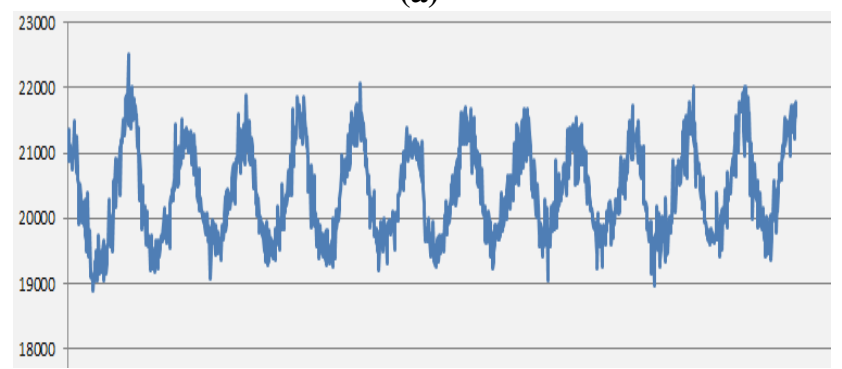

(b)

Fig 10(a) Deep breathe (b) Normal adult breathe.

Figure 10 shows the normal respiratory peaks measured for a minute for an adult. There are 13peaks showing the respiration rate. The normal respiration rate measurement for an adult is 12 to 18 breaths per minute. The research work can be viewed in two parts: In the first the prototype sound acquisition device is designed and ready for use. In second part impedance technique design and development of device has been discussed. Due to testing limitations and clinicians approval is awaiting for more robust tests. Presently few tests on voluntary normal adults as of interest with consented asthma patients with limited results are presented. The filing of $510(\mathrm{k})$ process, approval, and safety are major concern here since the involvement of power supply unit or battery operated connected directly on to body. Its non invasiveness might be easy to get certification and has to be certified.The primary classification of respiratory sound analyzer, impedance pnemogram and as well as end-tidal CO2 (ETCO2) categorized under Class-II in accordance with Food and Drug Administration (FDA) guidelines. Code for Federation of Regulation (CFR) provided a code $870.27 \mathrm{XX}$.

\section{FUTURE SCOPE OF THE WORK}

The research work has potential future scope in development of capnometer for pediatrics diagnostic device. This work can be further extended to detect apnea and hypoxia well before, child develops lack of oxygen. Non invasive $\mathrm{CO}_{2}$ end tidal monitoring of child patients. This is safeguarding and may be no doubt to becoming a benchmark diagnostic tool in monitoring pediatric patients.

\section{ACKNOWLEDGEMENT}

The authors would like to thank Management, Panchajanya Vidyapeetha Welfare Trust and Principal, Dr. Ambedkar Institute of Technology, Bangalore, India for their support and encouragement to carry continuum research work.

The authors would also like to thank DR.Subhash MN, Pediatrics post graduate student, Karnataka Institute of Medical Sciences, (KIMS), Hubballi, Karnataka, India and Vinu Rajakumar, reseach scholar, Vels Tech., Chennai, Tamilnadu, India for rendering their timely help in realizing this work

\section{REFERENCES}

1. S.D. Min, J.K. Kim, H.S. Shin, Y.H. Yun, C.K. Lee, and M. Lee:, "Noncontact Respiration Rate Measurement System Using an Ultrasonic Proximity Sensor," IEEE sensors journal, vol. 10, pp. 1732-1739, 2010.

2. Michael Ramsay, Respiratory Rate Acoustic Monitoring, Texas A\&M Health Science Center, Baylor Research Institute, Dallas, Texas, 2018.

3. Prabhu Rajkumar, Kamaraj Pattabi, Selaraj, Arvind Bhome, Mill Brashier, Prashanta Bhattacharya and Sanjay M Mehendale," A corss- sectional study on prevalence of chronic obstructive pulmonary disease (COPD) in India: rationale and methods" BMJ Open,pp.1-6,2017.

4. Jacome, Oliveira, Marques, "Computerized respiratory sounds: a comparison between patients with stable and exacerbated COPD", Clinincal Respiratory Journal, vol11, issue 5,pp.612-620, 2017.

5. Shih-Hong Li, Bor-Shing Lin 2, Chen-Han Tsai, ChengTa Yang,and Bor-Shyh Lin,"Design of Wearable Breathing Sound Monitoring System for Real-Time Wheeze Detection ,sensors ,vol.17, issue 171,pp.1$15,2017$.

6. C. Matiz C., N. Olarte L., and A. Rubiano F, "Electronic System Design for Respiratory Signal Processing", World Academy of Science, Engineering and Technology International Journal of Electronics and Communication Engineering, Vol.6, No.11, 2012.

7. Robert C. Beckerman and Michael 1. Wegmann, "A Comparison of Tracheal Breath Sounds, Airflow, and Impedance Pneumography in the Detection of Childhood Apnea," Journal of Sleep, vol. VIII, issue 4, Raven Press, New York, pp.342-346,1985.

8. Jo Rae Wright, Gerald Turino, James Sheller, Judy Neubauer, Steven Kunkel, Karen Fulton, Norman Edelman, David Earle, Nancy Dunlap and Christine Cannon, "Future Directions for Research on Diseases of the Lung," American Thoracic Society, Lung Association, American Journal of Respiratory and Critical Care Medicine, vol.158, pp.320-335,1998.

9. L Pekka Malmberg, Leena Pesu, Anssi R A Sovijarvi, "Significant differences in flow standardised breath sound spectra in patients with chronic obstructive pulmonary disease, stable asthma, and healthy lungs", Journal of Thorax vol.5,pp.1285-1291,1995.

10. Kentaro Ouchi, "Noninvasive Acoustic Respiration Rate Monitoring," SOJ Anesthesiology and Pain Management, vol3, issue 1,pp. 1-3,2016.

11. Amit K. Gupta, "Respiration Rate Measurement Based on Impedance Pneumography", Texas Instruments Incorporated, pp.1-10,2011.

12. K.V. Madhav, M.R. Ram, E.H. Krishna, N.R. Komalla, K.A. Reddy, "Estimation of Respiration Rate from ECG, BP and PPG signals using Empirical Mode Decomposition,” IEEE Instrumentation and 
Measurement Technology, pp.1-4, 2011.

13. Joint Commission on Accreditation of Healthcare Organizations. Sentinel event alert: patient controlled analgesia by proxy; JCAHO. 2004.

14. World Health Organization. World Health Statistics 2011, last accessed April 2012. www.who.int/whosis/whostat/2011/en/index.html.

15. Sanna Eriksson \& Sofia Isaksson, "Design and development of a medical Device for diagnosis of COPD," Division of Machine Design Department of Design Sciences Faculty of Engineering LTH, Lund University, pp.1-111, 2015.

16. Richard C.Fries, "Reliable Design of medical Device," CRC Press, 3rd Edition, 2017.

\section{AUTHORS PROFILE}

D.K. Ravish, Department of Medical Electronics, Dr.Ambedkar Institute of Technology, Bangalore, INDIA. Email: ravish_ait@yahoo.co.in

K.J.Shanthi, Department of Medical Electronics, Dr.Ambedkar Institute of Technology, Bangalore, INDIA. Email: shanti kj@yahoo.co.in

Nayana R Shenoy Department of Medical Electronics, Dr.Ambedkar Institute of Technology, Bangalore, INDIA. Email: nayana.rshenoy@gmail.com 